\title{
D. Rosenthal
}

\section{Endoscopic approaches to the thoracic spine}

Received: 23 November 1999

Accepted: 27 November 1999

\section{Rosenthal}

Neurochirurgische Praxisgemeinschaft, und Kliniken des Hochtaunuskreises, Bad Homburg v. d. Hoehe,

Hessenring 128,

61348 Bad Homburg v. d. Hoehe,

\section{Germany}

e-mail: Trosen@aol.com,

Tel: +49-6172-920464,

Fax: +49 6172-920469

\begin{abstract}
Endoscopic surgery of the thoracic spine has up to now been considered as an experimental procedure. Reports published in recent years have shown that the results achieved with this technique are as good as, or for some indications superior to, those reported for classic open approaches. A review of the indications, limitations, advantages and disadvantages is presented. Although
\end{abstract}

there is still resistance to acknowledging the effectiveness of this procedure, experience has shown that the results are as good, complications are fewer and postoperative recovery is improved, thus shortening the total hospitalization time.

Key words Endoscopic spine surgery $\cdot$ Thoracoscopic spine surgery $\cdot$ Video-assisted thoracoscopy

\section{Introduction}

During the last decades, advances in surgical equipment and refinement of surgical techniques have steadily increased the role of endoscopy in all fields of surgery. At the beginning of the 1990s, thoracoscopic procedures were extensively reported by cardiothoracic surgeons in the treatment of a variety of affections that involved the thoracic cavity $[2,9,10,13]$. Following adaptation of the equipment and refinement of the technique, and as a natural evolution of this trend, the first reports dealing with endoscopic spine surgery of the thoracic spine were published at the beginning of the 1990s [6, 14-16]. Because these approaches make use of endoscopic instruments, they gained popularity under the term "minimally invasive spine surgery".

\section{Is endoscopic spine surgery a "minimally invasive" technique?}

The definition of minimally invasive surgery, first published by Wickham and Fitzpatrick in 1990, is: "to reduce the physical trauma inflicted upon the patient to a minimum while achieving a maximum therapeutic result" [8].
But isn't this the purpose of all surgery? Surgical decisions are taken on the basis of balancing the "healing" and "harming" potential of a given treatment. Based on their judgement and expertise, physicians must always maximize the first and minimize the second, aiming to improve outcome. However, a simple reduction in the size of the skin incision is considered in many cases a "minimally invasive" procedure, which is not necessarily the correct interpretation of Wickham and Fitzpatrick's concept.

Obtaining or regaining stability has turned into one of the most important aspects of modern spinal surgery. Posterior and posterolateral techniques traumatize the structures involved in stability (muscles, ligaments, articular processes, etc.) to a higher degree than anterior or anterolateral approaches (Table 1). However, surgeons are still reluctant to use an anterior approach, arguing either that a second team of surgeons may be needed or that the risk of perioperative morbidity is higher, resulting in longer hospitalization time or that the opening of the pleura may cause severe complications [21]. Endoscopic surgery of the dorsal spine was developed to overcome those drawbacks, offering an alternative to the spine surgeon. By changing not only the size of the wound, but also the side of the access, from a posterior to an anterior one, spinal endoscopy has not only reduced the wound surface but also abolished completely trauma to structures that are of utmost impor- 
Table 1 Reasons to prefer an anterior approach

Access the pathology in a more direct fashion (anterior affections are approached anteriorly)

Keep the integrity of posterior structures

The natural (thorax) or virtual (retroperitoneum) cavities needed to gain enough working space are located anteriorly

Less tissue dissection (smaller access) is needed to reach these cavities (no firm muscle attachment as at the back)

Since the cavities are unfolded with minimal or no retraction at all, the endoscope can maximize its capacity of providing superb illumination and visualization in deep-seated areas in a gasless and waterless fashion

tance for stability. If we adapt the concept of minimal invasiveness to spine surgery, the definition would be: "to reduce the physical trauma inflicted upon the patient and on structures related to spinal stability to a minimum while achieving a maximum therapeutic result".

Because endoscopic spine surgery is based on a totally different philosophy of approaching spinal diseases, the term that fits best for these approaches is "less invasive" or "minimally incisional" surgery (LISS or MISS).

\section{Technical details and surgical steps}

Because a detailed description of the surgical steps for each indication would "burst the frame" for this chapter, only the technical aspects common to any thoracoscopic spinal access will be addressed.

\section{Operating room setup and patient positioning}

Surgery is performed in a standard operating room, with the patient under general anesthesia. If a stabilization is planned, the operating table used should be radiolucent, allowing fluoroscopic control to be performed throughout the surgery. A double-lumen endotracheal tube, inserted under endoscopic guidance, is used to achieve selective lung collapse, uncovering the spine and allowing the surgeon to approach the affected spinal segment. Under normal circumstances, the iatrogenic pneumothorax is enough to allow free access to the spine, avoiding prolonged retraction of the parenchyma. The patient is positioned in a stable lateral decubitus, with the side where the pathology is located facing upwards. If instrumentation is expected to be used above T9, the right side of the spine offers more working space between the head of the ribs and the acygos vein than the left side (aorta). Below T9, the aorta is located more anteriorly, so that the diaphragm can be pushed downwards and instrumentation can be performed with no major problem.

Positioning of the surgeons, scrub nurse and anesthetist are schematically drawn in Fig. 1.

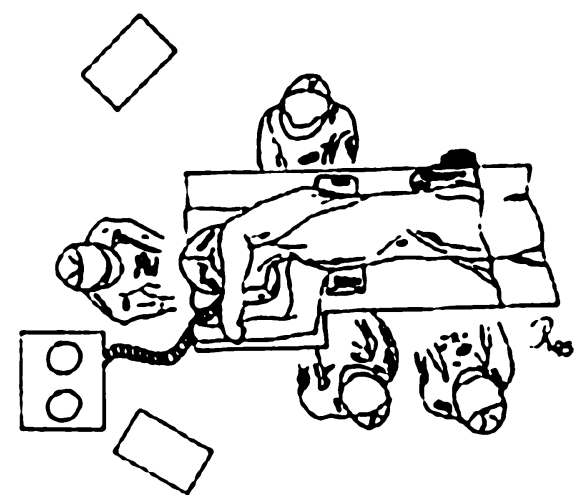

Fig. 1 The surgeon and instrumenting nurse stand in front, the assistant at the back and the anesthetist, cranial to the patient

\section{Instruments}

Specially designed instruments have a minimum length of $30 \mathrm{~cm}$ a width of between 0.3 and $1 \mathrm{~cm}$. A variety of scissors, graspers, retractors, rongeurs, curettes and dissectors have been adapted to fulfil technical requirements (Fig. 2A). MET is a gasless procedure utilizing a wide natural cavity (hemithorax), giving the surgeon clear access to the thoracic spine similar to thoracotomy. Hemostasis, can be a

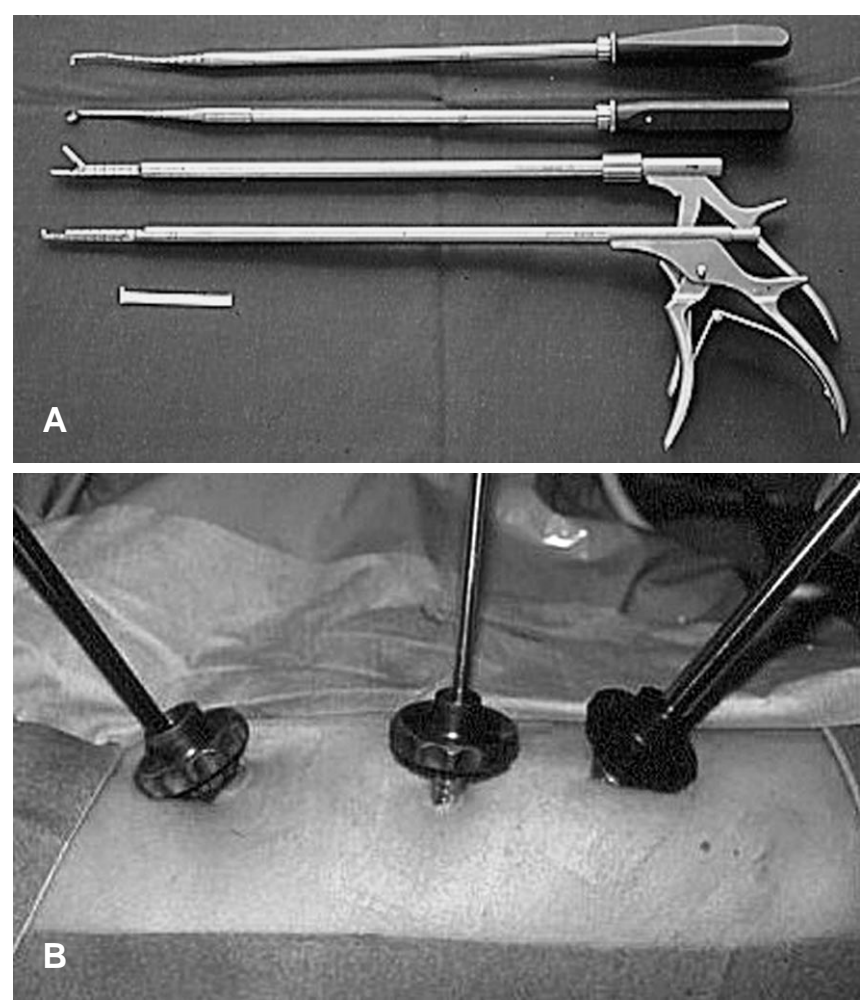

Fig. 2 A Selection of instruments used for thoracoscopic spine surgery. B The instruments enter the thoracic cavity through the previously placed trocars 

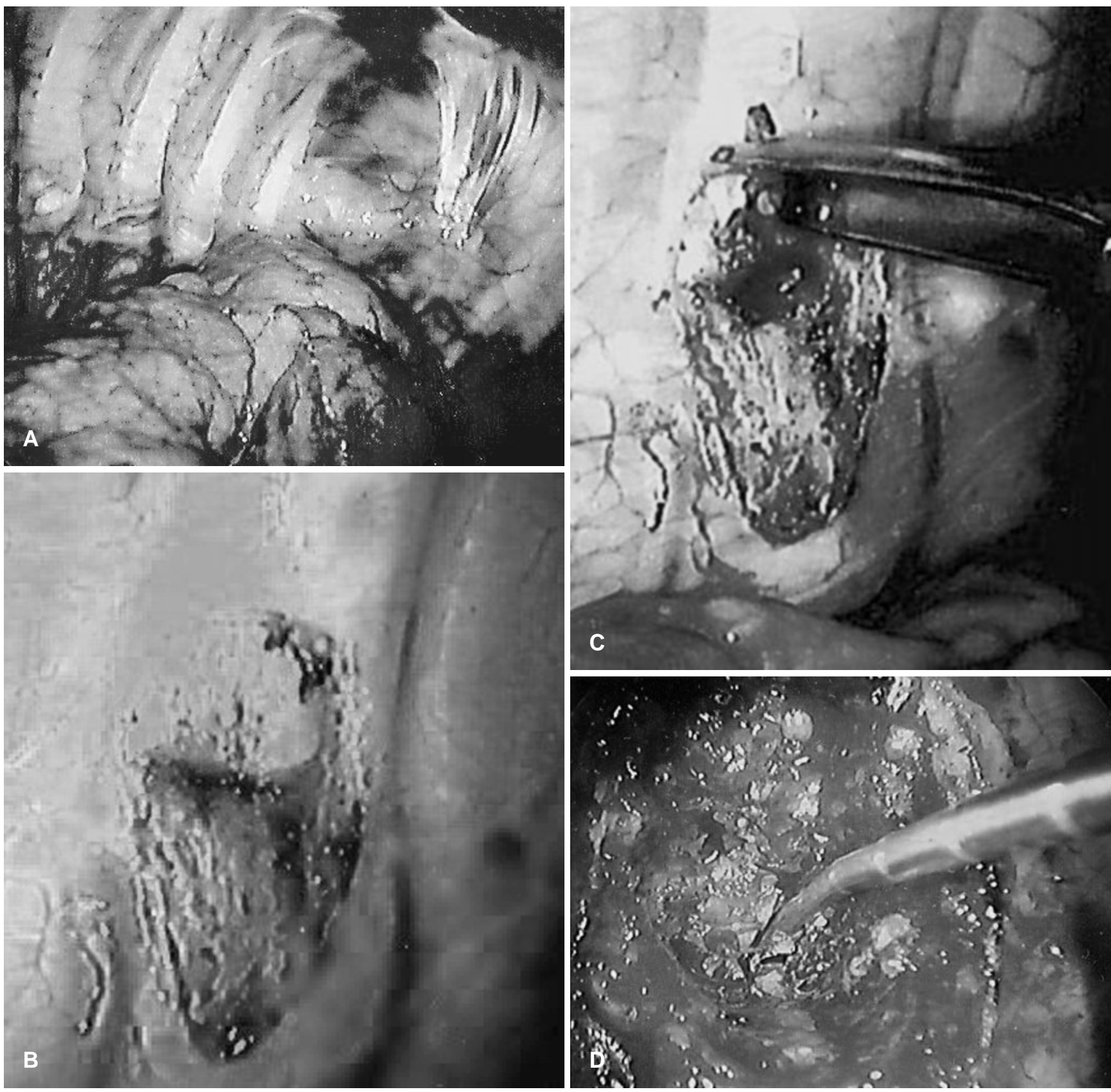

Fig. 3 A View of the thoracic cavity when entering with the endoscope. The lung is collapsed (iatrogenic pneumothorax) and uncovers the right side of the spine. B The rib head and the anterolateral portion of the disc space can be clearly recognized after splitting the parietal pleura. C The rib head is resected with an oscillating saw. D The spinal canal is free, the dural sac and the disc space can be clearly recognized

problem if the surgeon does not have the appropriate instruments. A variety of bipolar forceps have been designed, allowing the surgeon to coagulate epidural veins or arteries, avoiding the use of high-frequency diathermy (monopolar) at this site. A normal video chain (monitor, camera, light source and VCR), as used for arthroscopy or laparoscopy, is used. A rigid rod-lens scope, $10 \mathrm{~mm}$ in diameter with bidimensional imaging, and a $30^{\circ}$ angle optic is used. This allows the surgeon not only to focus on the operation field from different angles, but also avoids "fencing" with other instruments, which often happens when $0^{\circ}$ scopes are used. A table-mounted scope holder can be used to maintain the endoscope in a stable (and adjustable) position throughout the surgery. 
Fig. 4 A Magnetic resonance (MR) image shows the spaceoccupying lesion at T9-10 B Computed tomographic (CT) scan confirms a calcified thoracic disc herniation. C Postoperative MR image showing complete decompression of the spinal cord. D The postoperative CT scan shows the operative defect necessary to reach and remove the calcified hernia
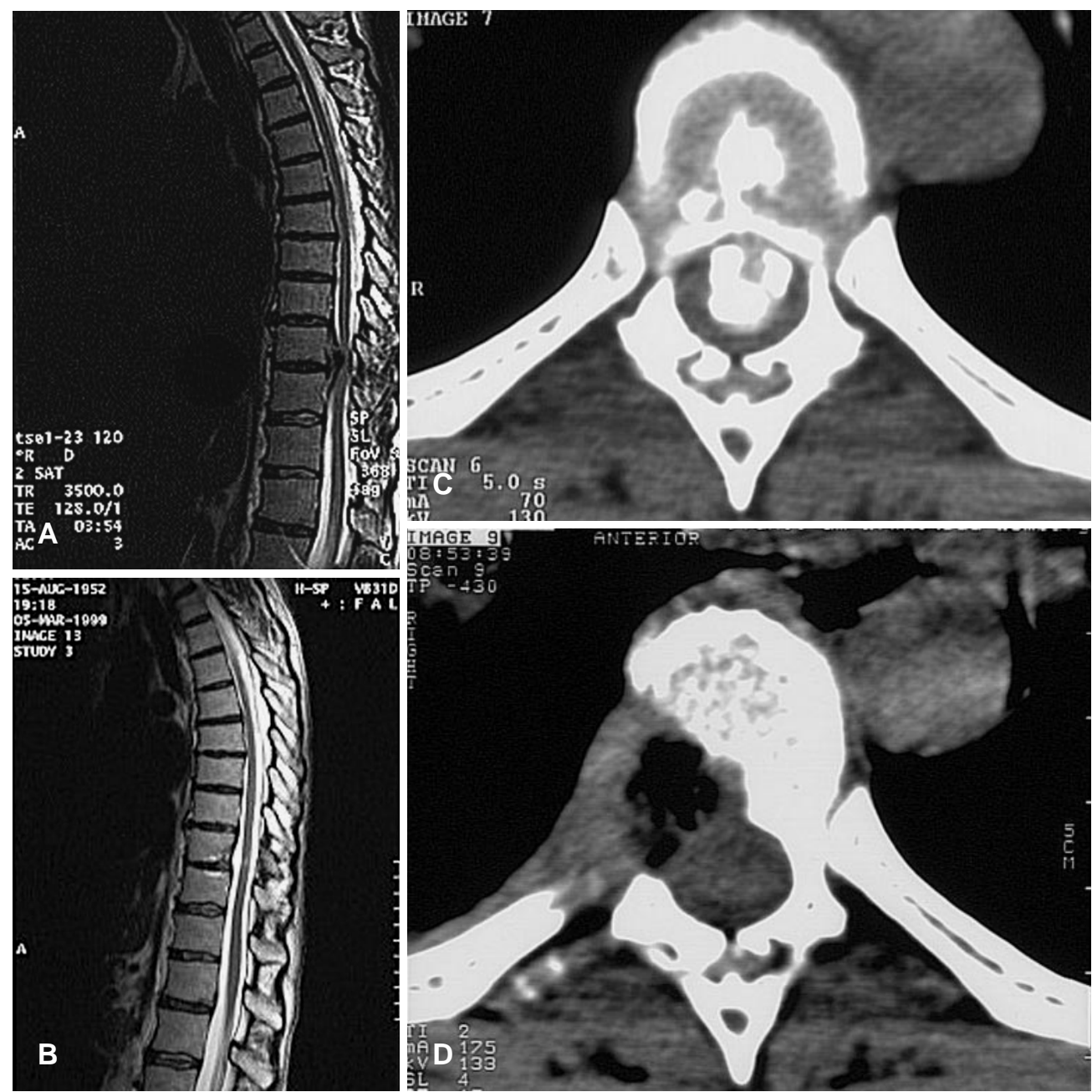

Portal placement and spinal access

Flexible silicon trocars with diameters ranging from 0.5 to $12 \mathrm{~cm}$ are used. By doing so, compression and irritation of the intercostal nerve is avoided; further advantages are that it is radiolucent (avoiding interference with fluoroscopy) and it permits curved instruments to be introduced into the chest. The trocars' insertion device is blunt and open, allowing the free passage of air in and out of the thoracic cavity (Fig. 2B). The placement of the first port is the most dangerous one, since it is introduced without visual control. It is usually positioned at the fifth or sixth intercostal space, between the middle and posterior axillary line. The second port is placed according to the location of the pathology, either above or below the first one. The working ports should be well apart from each other, allowing the instruments to converge towards a given point at the spine (triangulation). By doing so, "fencing" of the instruments, while spinal dissection is performed, can be avoided. The third port is usually placed slightly ventral to the two others, along the anterior axillary line and more caudal (at the 9th or 10th intercostal space). This port is used for the endoscope, allowing it to be placed above, below or between the instruments.

To insert the port, a skin incision of about $1.5 \mathrm{~cm}$ is made, parallel to the superior margin of the rib. Stump dissection of the subcutaneous tissue, intercostal muscles and parietal pleura are performed using a tissue clamp. Single lung ventilation should be started with the skin incision, in order to avoid mechanical lung injuries when opening the pleura.

Rotating the table about $30^{\circ}$ anteriorly will displace the lung and the mediastinum further up front, uncovering even more the anterolateral side of the spine.

Exposure of the spine and resection of the affected structures

Localization of the affected level is achieved by counting the heads of the ribs. Under normal circumstances the first rib is very difficult to visualize, but can be easily palpated (Fig. 3A). The second rib articulates at the foramen between T2 and 3, leading to the intervertebral disc. From 
Fig. 5 A, B MR image showing a dumbell neuroma at the T9-10 level. with intraspinal and intrathoracic extension. C, D Postoperative MR images (1 year after surgery), showing complete removal of the tumor. The patient returned to normal activity 6 weeks after surgery

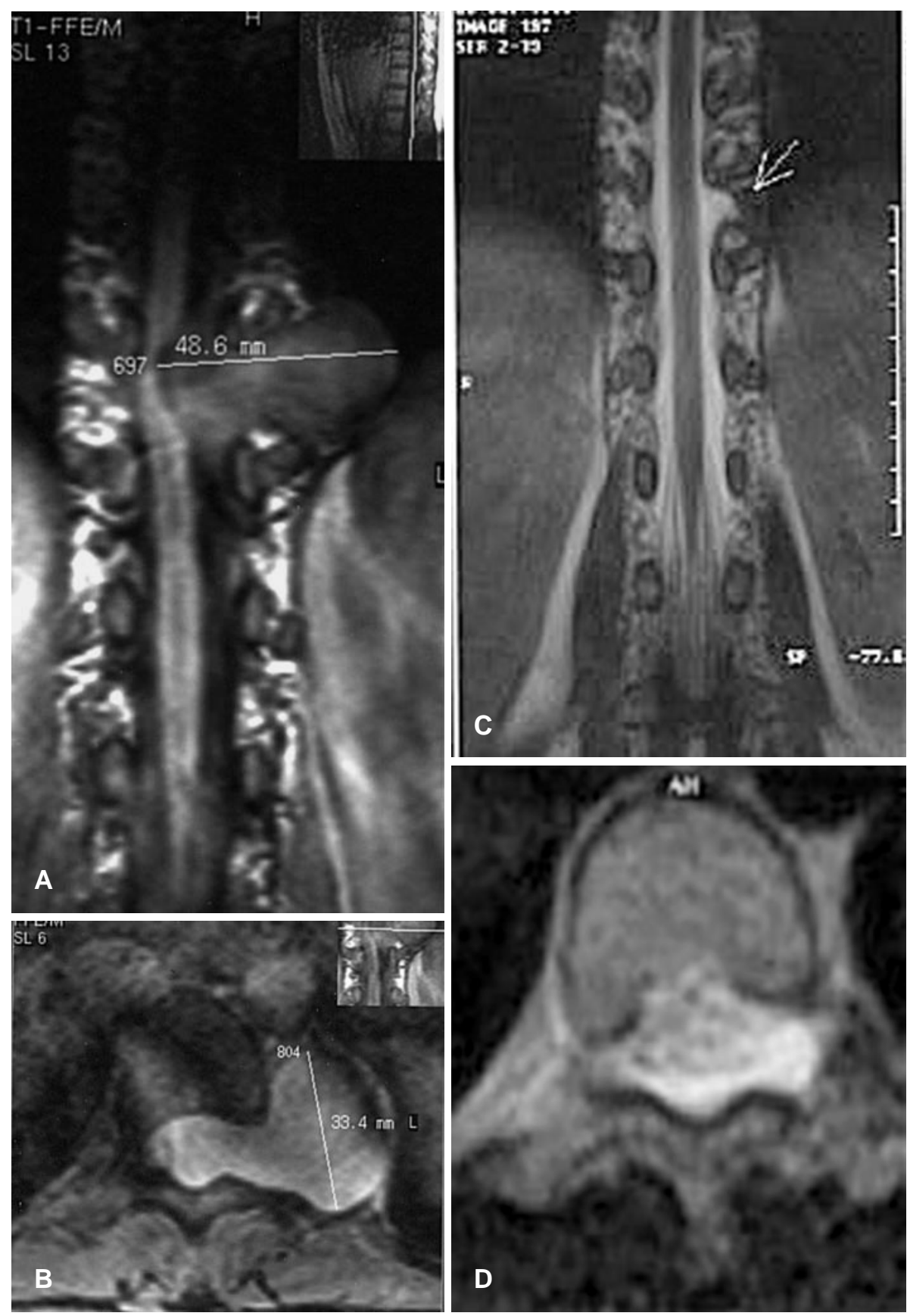

T2 down to T10, the head of the rib will always lead the surgeon to the disc, situated between two segmental vessels. When a tumor or a fracture alters the normal contours of the pleura or causes a hematoma, localization is even easier. Definite orientation and documentation is achieved by intraoperative fluoroscopy.

Dissection starts at the parietal pleura, which is opened with endoscopic scissors and high-frequency coagulation. Care has to be taken to start this maneuver at the head or the body of the rib, in order to avoid the segmental vessels that run above and below the disc space. Under normal circumstances the segmental vessels are kept intact. Since the approach to the spinal canal is done through the intervertebral foramen, usually there is no need to coagulate or clip the segmental vessels unless a vertebrectomy is planned. If, for example, T8 has to be removed, the surgeon has to ligate the segmental vessels of T8, followed by $\mathrm{T} 7$ and $\mathrm{T} 9$ (where the instrumentation is attached to the spine) (Fig. 3B).

The next step is the resection of the head of the rib and the pedicle so that immediate control of the foramen and spinal canal is achieved. In cases where the head of the rib 
Fig. 6A, B Metastatic tumor at T7-8, with compromise of posterior structures, intraspinal invasion and spinal cord compression. C, D Postoperative plain radiographs showing $360^{\circ}$ decompression and reconstruction. The ventral resection and stabilization was performed using the endoscopic technique.
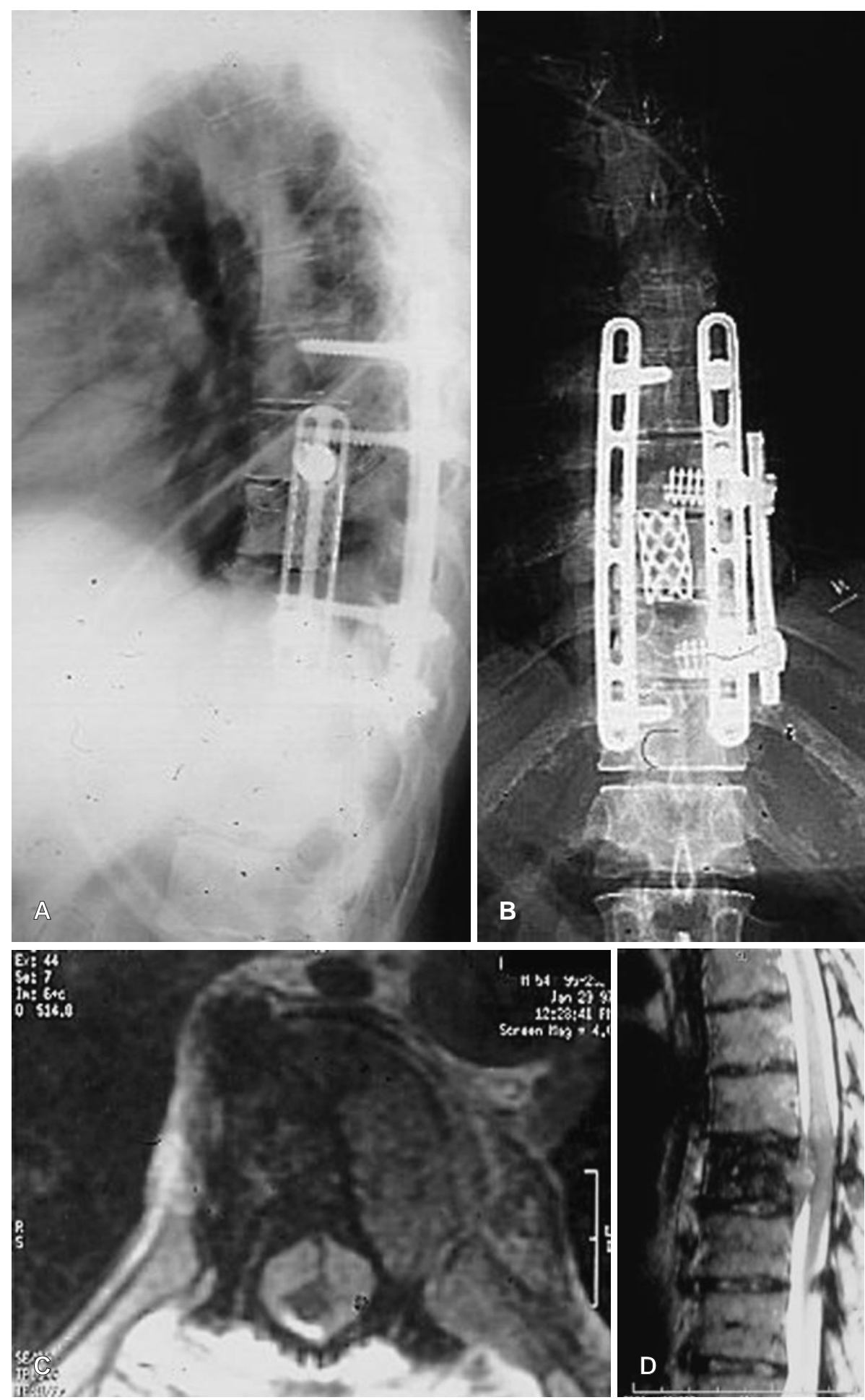

has already been destroyed by the underlying process, careful removal of soft tissue will uncover the foramen and give access to the spinal canal. Care has to be taken not to enter the spinal canal with big instruments (rongeurs or punches) at this stage. When mass-occupying le- sions are compressing the spinal cord (herniated disc, tumor or bone) the instrument increases pressure on the medulla and may cause irreparable damage. To create a safe working area, the surgeon removes the anterior boundaries of the foramen (posterior margin of the vertebral 
bodies and disc), and if this is not enough, part of the pedicle of the vertebra below (Fig. 3C-D).

After following these steps, the spinal canal is exposed and mass-occupying lesions can be removed. The surgeon always moves his instruments from the spinal canal to the chest cavity, avoiding working in the opposite direction, since a slippage with the curette or rongeur would directly hit the dural sac. When the occupying lesion is too big, piecemeal resection is preferred, starting at the base of the mass, and leading to a gradual mobilization of the process towards the ventral part of the canal, where it can be removed.

\section{Indications}

The list of procedures that can be accomplished at present is summarized in Table 2.

\section{Biopsy}

Biopsy of affections of the vertebral body, together with anterior release, are the two least demanding indications for thoracoscopy. The approach is straightforward and uncomplicated. It provides direct visualization of the affected segment and exact location of the probe. Due to the direct approach, endoscopic biopsy is superior to the needle biopsy, as it avoids spreading contaminated tissue throughout the punction canal.

\section{Anterior release for scoliosis}

Since 1993 a variety of papers have been published showing that endoscopically performed anterior release achieves the same degree of reliability as thoracotomy, achieving similar degrees of correction as releases performed through a thoracotomy.

The improved postoperative recovery (due to the reduction of trauma), better cosmesis and the reliability of the procedure with regard to achieving the discectomy, thus allowing better correction of the curvature, makes this one of the clearest and least controversial indications for endoscopy at present. Newly designed instrumentation devices enable the treatment of scoliosis with anterior

Table 2 Current indications for spinal endoscopy

\section{Biopsy}

Anterior release for deformities

Thoracic disc herniation

Neurogenic tumors with intrathoracic extension (schwannoma, neurofibroma)

Vertebrectomy, decompression, reconstruction and stabilization (tumor, trauma or infection) screws and bars. First experiences reported show promising results $[1,3,7]$.

Thoracic disc herniation

Since the first publication on this topic a lot of experience has been obtained in the treatment of thoracic disc herniation. Reports showing long-term results obtained after endoscopic discectomy $[17,19]$ or reoperation of discs previously treated with classic open techniques [5] confirmed the assumptions made in the original paper in 1994 [14]. Endoscopy not only achieves the same results as open thoracotomy [22], but also reduces tissue damage and complications. A comparison between thoracotomy, costotransversectomy and endoscopy has shown that, due to the marked reduction of trauma, endoscopy has lowered the percentage of complications, enhanced recovery and shortened hospitalization time.

Tumors originating from the nerve or nerve sheath with intrathoracic extension

The most common tumors in this area are schwannomas or neurofibromas. Although peripherally situated tumors are far more accessible to thoracoscopy, the technique also allows tumors with intra- and extracanalicular extension to be resected in a reliable and safe manner Since these tumors are very infrequent, there are only individual case reports presented in the literature [4, 12 ].

At present, tumors extending in and outside the spinal canal are not recommended for endoscopic resection, since a watertight dural closure may be difficult using endoscopy.

Vertebrectomy, reconstrucution and instrumentation

Affections of the anterior column caused by tumor, trauma or infection can be excised, reconstructed and stabilized using anterior instrumentation devices. The main advantage of endoscopy is that the upper thoracic spine (T2T4) and the thoracolumbar junction (T11-L2) can be approached with no disinsertion of the scapula or minimal disinsertion of the diaphragm, enabling complex reconstructions without additional trauma $[6,15,16]$.

\section{Contraindications}

Contraindications can be classified into unspecific (common to all surgical techniques) and specific (related only to endoscopic procedures). Although pulmonary restriction can be a problem, it should be considered as a relative contraindication since it has been shown that, although 
Table 3 Unspecific and specific contraindications for endoscopic procedures at the thoracic spine

Unspecific
Coagulation disturbances
Cardiovascular
Pulmonary
Others
Specific
Pachipleuritis
Pneumonectomy on the contralateral side of the approach
Severe pulmonary restriction
Selective intubation not possible

pulmonary function may be reduced at the beginning of surgery, it normalizes very rapidly, allowing the operation to procede normally [11].

Among the specific contraindications, pneumonectomy of the contralateral side of the approach and the impossibility of performing selective intubation are the most important and specific contraindications for this procedure (Table 3 ).

\section{Conclusion}

Since the first laboratory experiences in 1991, endoscopic spine surgery has experienced rapid developments, and now covers the whole spectrum of indications for anterior approaches.

It is a safe and reliable technique, with a high potential to reduce unnecessary trauma on soft tissue as well as on structures intimately related to stability, thus achieving the same results as an open approach. Complex procedures like vertebral resection, reconstruction and stabilization,
Table 4 Advantages of thoracoscopic spine surgery

Reduces trauma on structures related to stability (muscles,
ligaments, articular processes) and soft tissue
Multiple levels can be reached through the same ports
Because of a significant reduction in postoperative stress
(less pain, less blood loss, improved respiratory function),
postoperative recovery is enhanced, reducing intensive care
unit and total hospitalization time
Improved visualization and illumination allowing the surgeon
to change rapidly between macro and microsurgical pictures
Ideal visualization of the cervicothoracic and thoracolumbar spine
without need to disinsert the scapula or diaphragm

Table 5 Disadvantages of thoracoscopic spine surgery

Learning curve

Anatomy and topography may disorient (due to magnification)

Image distortion, lack of tridimensional images

Development of instruments and instrumentation just started

or correction of spinal deformities, can be treated using this technique as well (Table 4). Although the basic anatomy and dissection techniques are familiar to most spinal surgeons, the use of longer tools, as well as the new ways of visualization, perception and spatial orientation have to be learned at the laboratory before starting clinical application (Table 5).

At the beginning of the 1990s, endoscopic spine surgery at the thoracic spine was considered as an "experimental procedure", quickly turning into a "gimmick" or a "possibility" in the last years. At the start of the new millenium, thoracoscopic spine surgery has to be considered as an "alternative" and probably as a "routine" procedure for future generations of spine surgeons.

\section{References}

1. Blackman RG, Luque E (1999) Endoscopic anterior correction of idiopathic scoliosis. In: Dickman CA, Rosenthal DJ, Perin NI (eds) Thoracoscopic spine surgery. Thieme, New York, pp 189217

2. Coltharp WH, Arnold WC Jr, Burrus GR, Glassford DM Jr, Lea JW IV, Petracek MR, Starkey TD, Stoney WS, Thomas CS Jr, Sadler RN (1992) Videothoracoscopy: improved technique and expanded indications. Ann Thorac Surg 53: 776-779

3. Crawford AH (1999): Anterior release of spinal deformities, In: Dickman CA, Rosenthal DJ, Perin NI (eds) Thoracoscopic spine surgery. Thieme, New York, pp 169-188
4. Dickman C, Apfelbaum R (1998) Thoracoscopic microsurgical excision of a thoracic schwannoma. Neurosurg Focus 4: Article 5

5. Dickman C, Rosenthal D, Regan J (1999) Reoperation for herniated thoracic discs. Neurosurg Focus 6: Article 5

6. Dickman CA, Rosenthal D, Karahalios D, Paramore CG, Mican CA, Apostolides PJ, Lorenz R, Sonntag VKH (1996) Thoracic vertebrectomy and reconstruction using a microsurgical thoracoscopic approach. Neurosurgrey 38: 279-293

7. Dickman CA, Mican CA (1996) Multilevel anterior thoracic discectomies and anterior interbody fusion using a microsurgical thoracoscopic approach. Case report. J Neurosurg 84: 104-109
8. Fitzpatrick JM, Wickham JEA (1990) Minimal invasive surgery. Br J Surg. 77: 721-722

9. Kaiser LR (1994) Video-assisted thoracic surgery: current state of the art. Ann Surg 220: 720-734

10. Landreneau RJ, Mack MJ, Hazelrigg SR, Dowling RD, Acuff TE, Magee MJ, Ferson PF (1992) Video-assisted thoracic surgery. Basic technical concepts and intercostal approach strategies. Ann Thorac Surg 54: 800-807

11. Lischke V, Westphal K, Behne M, Wilke HJ, Rosenthal D, Marquardt G, Kessler P (1998) Thoracoscopic microsurgical technique for vertebral surgery-anesthetic considerations. Acta Anesthesiol Scand 42: 1199-1204 
12. Lyons MK, Gharagozloo F (1995) Video assisted throacoscopic resection of intercostal neurofibroma. Surg Neurol 43: 542-545

13. Mack MJ, Aronoff RJ, Acuff TE, Douthit MB, Bowman RT, Ryan WH (1992) Present role of thoracoscopy in the diagnosis and treatment of diseases of the chest. Ann Thorac Surg 54: 403-409

14. Rosenthal D, Rosenthal R, De Simone A (1994) Removal of a protruded thoracic disc using microsurgical endoscopy. A new technique. Spine 19: 1087-1091

15. Rosenthal D (1995): Newer application of spinal instrumentation. Endoscopic internal fixation of the thoracic spine. In: Regan JJ, McAfee PC, Mack MJ (eds) Atlas of endoscopic spine surgery. Quality Medical Publishing, St. Louis, pp 333-337
16. Rosenthal D, Marquardt G, Lorenz R, Nichtweiß M (1996) Anterior decompression and stabilization using a microsurgical endoscopic technique for metastatic tumors of the thoracic spine. J Neurosurg 84: 565-572

17. Rosenthal D, Dickman C, Lorenz R, et.al (1996) Thoracic disc herniation: early results after surgical treatment using microsurgical endoscopy (abstract). J Neurosurg 84: 334 A

18. Rosenthal D, Paolucci V, Yahya H, Maddalozzo L, Lorenz R (1997) Microsurgical endoscopic-assisted retroperitoneal approach to the thoracolumbar and lumbar spine. Tech Neurosurg 3: 315-321

19. Rosenthal D, Dickman C (1998) Thoracoscopic microsurgical excision of herniated thoracic discs. J Neurosurg 89: 224-235

20. Rosenthal D, Dickman C (1999) Thoracoscopic microsurgical excision of herniated thoracic discs. Neurosurg Focus 6: Article 4
21. Shaw B, Mansfield FL, Borges L (1989) One stage posterolateral decompression and stabilization for primary and metastatic vertebral tumors in the thoracic and lumbar spine. J Neurosurg 70: 405-410

22. Shekar L, Jannetta PJ (1983) Thoracic disc herniation: operative approaches and results. Neurosurgery 12: 303-305

23. Stillerman CB, Chen TC, Couldwell WT, Zhang W, Weiss MH (1998) Experience in the surgical management of 82 symptomatic herniated thoracic discs and review of the literature. J Neurosurg 88: 623-633 